\title{
Mechanisms of Non-Fatal Stent-Related Myocardial Infarction Late Following Coronary Stenting With Drug-Eluting Stents and Bare Metal Stents - Insights From Optical Coherence Tomography -
}

\author{
Periklis A. Davlouros, MD, PhD; Vasileios Karantalis, MD; Ioanna Xanthopoulou, MD; \\ Eleni Mavronasiou, MD; Grigorios Tsigkas, MD; \\ Konstantinos Toutouzas, MD, PhD; Dimitrios Alexopoulos, MD, PhD
}

\begin{abstract}
Background: A prospective observational study using optical coherence tomography (OCT) of patients with myocardial infarction (MI), late following drug-eluting (DES) or bare metal stent (BMS) implantation, when the stented segment was considered culprit.

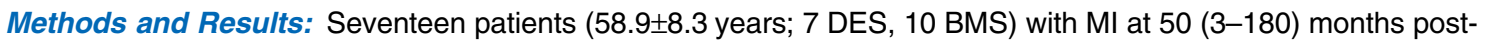
stenting. Patients with BMS sustained a MI later than patients with DES (95 (3-180) vs. 8 (3-62) months, $P=0.01$ ]; $5(71.4 \%)$ of the DES patients demonstrated binary angiographic restenosis, in contrast to $8(80 \%)$ with BMS $(P=1.0)$. DES had significantly less thickness of the neointimal hyperplasia compared with BMS $(0.08 \pm 0.04$ vs. $0.36 \pm 0.2 \mathrm{~mm}$, $P=0.003)$. None of the DES was totally covered with neointimal tissue. The overall percentage of uncovered and malapposed struts (ANCOVA), was significantly higher in DES than BMS (1.96, 95\% confidence interval (CI) 1.5-2.4 vs. $0.25,95 \% \mathrm{Cl}-0.1-0.6, \mathrm{P}<0.001$, and $0.66,95 \% \mathrm{Cl} 0.29-1.03$ vs. $0.11,95 \% \mathrm{Cl}-0.19-0.4, \mathrm{P}=0.03$, respectively). OCT features of atherosclerosis (lipid, neovascularization, or calcification) and possible neointimal rupture were found only in patients with BMS. Thrombus detection was not different between the 2 groups.
\end{abstract}

Conclusions: Stent-related, non-fatal, late acute Ml following stent implantation occurs later in patients with a BMS compared with those with a DES, and the mechanism includes delayed healing (mainly DES), and neointimal hyperplasia with atherosclerotic transformation and subsequent rupture (mainly BMS). (Circ J 2011; 75: 27892797)

Key Words: Bare metal stent; Drug-eluting stent; Myocardial infarction; Optical coherence tomography

A cute myocardial infarction (MI) occurring late following stenting may be related to either the stent or disease progression at the same or other coronary arteries. ${ }^{1}$ Stent-related MI involves stent thrombosis, with or without restenosis. Pathologic and intravascular ultrasound studies have shown that delayed healing with incomplete neointimal tissue strut coverage, vessel remodeling, and inflammation constitutes the most frequent substrate of late and very late drug-eluting stent (DES) thrombosis (LST)..$^{2-5}$ Prospective angioscopic and optical coherence tomography (OCT) studies have shown that features possibly associated with DES-LST, such as uncovered stent struts, may be found very frequently, even late post-stenting, depending on the type of DES. ${ }^{6-10}$ However, the reported rate of clinical LST is much lower than the reported rate of uncovered DES struts, underlining the fact that this phenomenon is multifactorial and questioning the relevance of the abovementioned OCT findings in prospective studies without clinical events.

On the other hand, LST also happens following bare metal stent (BMS) implantation and may have different chronology and mechanisms, as delayed healing is not an issue with these stents. ${ }^{4,11,12}$ Very recently, some case series and studies using intracoronary imaging in patients with late clinical events following BMS implantation are suggesting that development of new atherosclerotic lesions inside the BMS may be a possible mechanism explaining late events. ${ }^{13-16}$ More intriguing is the fact that features possibly indicative of neointimal "atherosclerotic transformation" have been found also inside DES and questions exist regarding its possible role in LST. $6,12,17$

Received June 6, 2011; revised manuscript received July 6, 2011; accepted July 26, 2011; released online September 14, 2011 Time for primary review: 20 days

Patras University Hospital, Patras (P.A.D., V.K., I.X., E.M., G.T., D.A.); University of Athens Medical School, Athens (K.T.), Greece

Mailing address: Vasileios Karantalis, MD, Patras University Hospital, Rion, Patras, Greece. E-mail: vkarantalis@upatras.gr

ISSN-1346-9843 doi:10.1253/circj.CJ-11-0581

All rights are reserved to the Japanese Circulation Society. For permissions, please e-mail: cj@j-circ.or.jp 


\begin{tabular}{lccc} 
Table 1. Baseline Characteristics of the Patients & & & \\
& DES $(\mathbf{n}=\mathbf{7})$ & BMS $(\mathbf{n}=10)$ & P value \\
Age (years) & $59.1 \pm 8.8$ & $58.7 \pm 8.4$ & 0.9 \\
Male & $5(71.4)$ & $10(100)$ & 0.2 \\
Diabetes mellitus & $3(42.9)$ & $3(30.0)$ & 0.6 \\
Hypertension & $5(71.4)$ & $6(60.0)$ & 1.0 \\
Hypercholesterolemia & $4(57.1)$ & $6(60.0)$ & 1.0 \\
Smoking & $2(28.6)$ & $6(60.0)$ & 0.3 \\
Family history of CAD & $0(0)$ & $0(0)$ & NA \\
Prior MI & $2(28.6)$ & $4(40.0)$ & 1.0 \\
Prior CABG & $0(0)$ & $2(20.0)$ & 0.5 \\
Initial clinical presentation & & & 0.5 \\
ACS & $7(100 \%)$ & $8(80 \%)$ & 0.1 \\
$\quad$ STEMI & $1(14.3)$ & $6(60.0)$ & 1.0 \\
$\quad$ NSTEMI & $1(14.3)$ & $1(10.0)$ & 0.04 \\
$\quad$ UA & $5(71.4)$ & $1(10.0)$ & 1.0 \\
SA & $0(0)$ & $1(10.0)$ & 1.0 \\
Provocativa ischemia & $0(0)$ & $1(10.0)$ & 1.0 \\
No. of vessels with significant disease & & & 0.6 \\
1 & $4(57.1)$ & $6(60.0)$ & 0.6 \\
2 & $1(14.3)$ & $3(30.0)$ & 1.0 \\
3 & $2(28.6)$ & $1(10.0)$ & $4(40.0)$ \\
Multivessel disease & $3(42.9)$ & & \\
\hline
\end{tabular}

Values are expressed as mean \pm SD or $n(\%)$. P-values derived from Student's t-test for continuous variables or Fisher's exact test for dichotomous variables.

DES, drug-eluting stent; BMS, bare metal stent; CAD, coronary artery disease; MI, myocardial infarction; CABG, coronary artery bypass grafting; ACS, acute coronary syndrome; STEMI, ST-elevation MI; NSTEMI, non-ST-elevation MI; UA, unstable angina; SA, stable angina.

\begin{tabular}{|lccc|}
\hline Table 2. Baseline Characteristics of the Lesions & & & \\
Lesion location & DES $(\mathbf{n}=\mathbf{7})$ & BMS $(\mathbf{n}=\mathbf{1 0})$ & P value \\
LAD & & & \\
LCX & $6(85.7)$ & $4(40.0)$ & 0.1 \\
RCA & $0(0)$ & $2(20.0)$ & 0.5 \\
Prox & $1(14.3)$ & $4(40.0)$ & 0.3 \\
Mid & $5(71.4)$ & $6(60.0)$ & 1.0 \\
Distal & $2(28.6)$ & $3(30.0)$ & 1.0 \\
Lesion morphology & $0(0)$ & $1(10.0)$ & 1.0 \\
A & & & NA \\
B1 & $0(0)$ & $0(0)$ & 0.2 \\
B2 & $0(0)$ & $3(30.0)$ & 1.0 \\
C & $5(71.4)$ & $7(70.0)$ & 0.2 \\
Stent length (mm) & $2(28.6)$ & $0(0)$ & 0.2 \\
Stent diameter (mm) & $21.6 \pm 8.4$ & $17.5 \pm 4.1$ & 0.3 \\
Total occlusion & $3.0 \pm 0.4$ & $3.2 \pm 0.2$ & 0.4 \\
Presence of thrombus & $1(14.3)$ & $0(0)$ & 1.0 \\
In-stent restenotic lesion & $2(28.6)$ & $2(20.0)$ & 0.4 \\
\hline
\end{tabular}

Values are expressed as means $\pm \mathrm{SD}$ or $\mathrm{n}(\%)$ for dichotomous variables.

LAD, left anterior descending artery; LCX, left circumflex artery; RCA, right coronary artery. Other abbreviations see in Table 1.

We therefore conducted an OCT study of all consecutive patients presenting with non-fatal acute MI beyond 1 month of DES or BMS implantation, in whom the stented segment was considered the culprit lesion, in an effort to identify the underlying possible mechanisms.

\section{Methods}

\section{Patient Population and Angiographic Data}

From October 2008 until May 2010, all consecutive patients with previous coronary stenting presenting with non-fatal acute MI at least 1 month post-stenting, and undergoing coronary angiography within $24 \mathrm{~h}$ in our centre were screened for inclu- 


\begin{tabular}{lccc|}
\hline Table 3. Follow-up Angiography During Non-Fatal Acute MI & & \\
& DES $(\mathbf{n}=\mathbf{7})$ & BMS (n=10) & P value \\
Interval (months) & $8(3-62)$ & $95(3-180)$ & 0.01 \\
STEMI & $3(42.9)$ & $7(70.0)$ & 0.4 \\
No. of vessels with significant disease & & & \\
1 & $4(57.1)$ & $3(30.0)$ & 0.4 \\
2 & $1(14.3)$ & $4(40.0)$ & 0.3 \\
3 & $2(28.6)$ & $3(30.0)$ & 1.0 \\
Lesion morphology & & & \\
A & $2(28.6)$ & $1(10.0)$ & 0.5 \\
B1 & $0(0)$ & $2(20.0)$ & 0.5 \\
B2 & $2(28.6)$ & $3(30.0)$ & 1.0 \\
C & $3(42.9)$ & $4(40.0)$ & 1.0 \\
Presence of thrombus & $5(71.4)$ & $7(70.0)$ & 1.0 \\
Total occlusion & $2(28.6)$ & $3(30.0)$ & 1.0 \\
\hline
\end{tabular}

Values are expressed as mean \pm SD or medians (range) for continuous variables or $n(\%)$ for dichotomous variables Abbreviations see in Table 1.

\begin{tabular}{|c|c|c|c|}
\hline OCT Parameters & DES $(n=7)$ & BMS $(n=10)$ & $P$ value \\
\hline Total struts per stent & $167.4 \pm 43.8$ & $226.2 \pm 141.9$ & 0.3 \\
\hline Uncovered struts per stent (\%) & $31.8 \pm 10.1$ & $3.66 \pm 6.1$ & $<0.001$ \\
\hline Malapposed struts per stent (\%) & $14.78 \pm 15.87$ & $1.57 \pm 3.9$ & 0.07 \\
\hline Malapposed and uncovered struts per stent (\%) & $9.55 \pm 11.0$ & $0.64 \pm 1.7$ & 0.08 \\
\hline Malapposition ratio (\%) & $33.25 \pm 22.3$ & $3.18 \pm 7.1$ & 0.01 \\
\hline Protruding struts per stent (\%) & $2.60 \pm 2.3$ & $0.48 \pm 1.0$ & 0.06 \\
\hline Total cross-sections with $>30 \%$ uncovered struts per stent & $43.3 \pm 27.5$ & $3.53 \pm 8.9$ & 0.008 \\
\hline Mean NIH thickness (mm) & $0.08 \pm 0.04$ & $0.36 \pm 0.2$ & 0.003 \\
\hline $\mathrm{NIH}$ area $\left(\mathrm{mm}^{2}\right)$ & $0.96 \pm 0.4$ & $3.92 \pm 1.7$ & $<0.001$ \\
\hline $\mathrm{NIH}$ area $(\%)$ & $11.68 \pm 3.6$ & $49.74 \pm 15.7$ & $<0.001$ \\
\hline Binary strut coverage (\%) & $16.08 \pm 18.4$ & $90.0 \pm 15.6$ & $<0.001$ \\
\hline Stent totally covered & $0(0)$ & $5(50.0)$ & 0.04 \\
\hline NUS & $2.18 \pm 0.3$ & $1.55 \pm 0.2$ & 0.001 \\
\hline Heterogeneity score & $1.85 \pm 0.7$ & $1.3 \pm 0.9$ & 0.2 \\
\hline SEI & $0.92 \pm 0.03$ & $0.93 \pm 0.02$ & 0.2 \\
\hline Fat & $0(0)$ & $6(60.0)$ & 0.04 \\
\hline Neovascularization & $0(0)$ & $4(40.0)$ & 0.1 \\
\hline Calcium & $0(0)$ & $3(30.0)$ & 0.2 \\
\hline Thrombus & $4(57.1)$ & $9(90.0)$ & 0.3 \\
\hline Rupture & $0(0)$ & $7(70.0)$ & 0.01 \\
\hline Mean stent area $\left(\mathrm{mm}^{2}\right)$ & $8.19 \pm 1.4$ & $7.95 \pm 2.18$ & 0.8 \\
\hline Mean lumen area $\left(\mathrm{mm}^{2}\right)$ & $7.22 \pm 1.1$ & $4.03 \pm 1.9$ & 0.001 \\
\hline Stent restenosis & $0(0)$ & $8(80.0)$ & 0.01 \\
\hline
\end{tabular}

Time-domain OCT used to assess 5 BMS and 3 DES and frequency-domain OCT of 5 BMS and 4 DES. Values are expressed as means \pm SD or $n(\%)$. P-values are from Student's $t$-test for continuous variables or Fisher's exact test for dichotomous variables.

OCT, optical coherence tomography; NIH, neointimal hyperplasia; NUS, neointimal unevenness score; SEI, stent eccentricity index. Other abbreviations see in Table 1.

sion in this study. When the culprit lesion was located at the previously stented segment of the vessel, the patient underwent OCT imaging of the latter, following informed consent. The previously stented lesion was considered culprit when significant angiographic stenosis ( $\geq 50 \%)$ and/or thrombosis was present, with absence of significant native lesions in the same or other coronary arteries. In the latter case, the stented vessel was considered culprit if there were evident ST-T changes on ECG in its corresponding perfusion territory during chest pain.
Demographics, medical history and clinical data were recorded. MI was defined as elevation of cardiac enzymes $>3$-fold the upper limit of normal and categorized as ST-segment elevation MI (STEMI) and non-STEMI (NSTEMI). In every patient, coronary angiography was performed via a femoral approach with standard catheters and techniques before OCT and angioplasty. If thrombus was evident on angiography, thromboaspiration was carried out before performing OCT.

Thrombus was defined as a filling defect during angiog- 


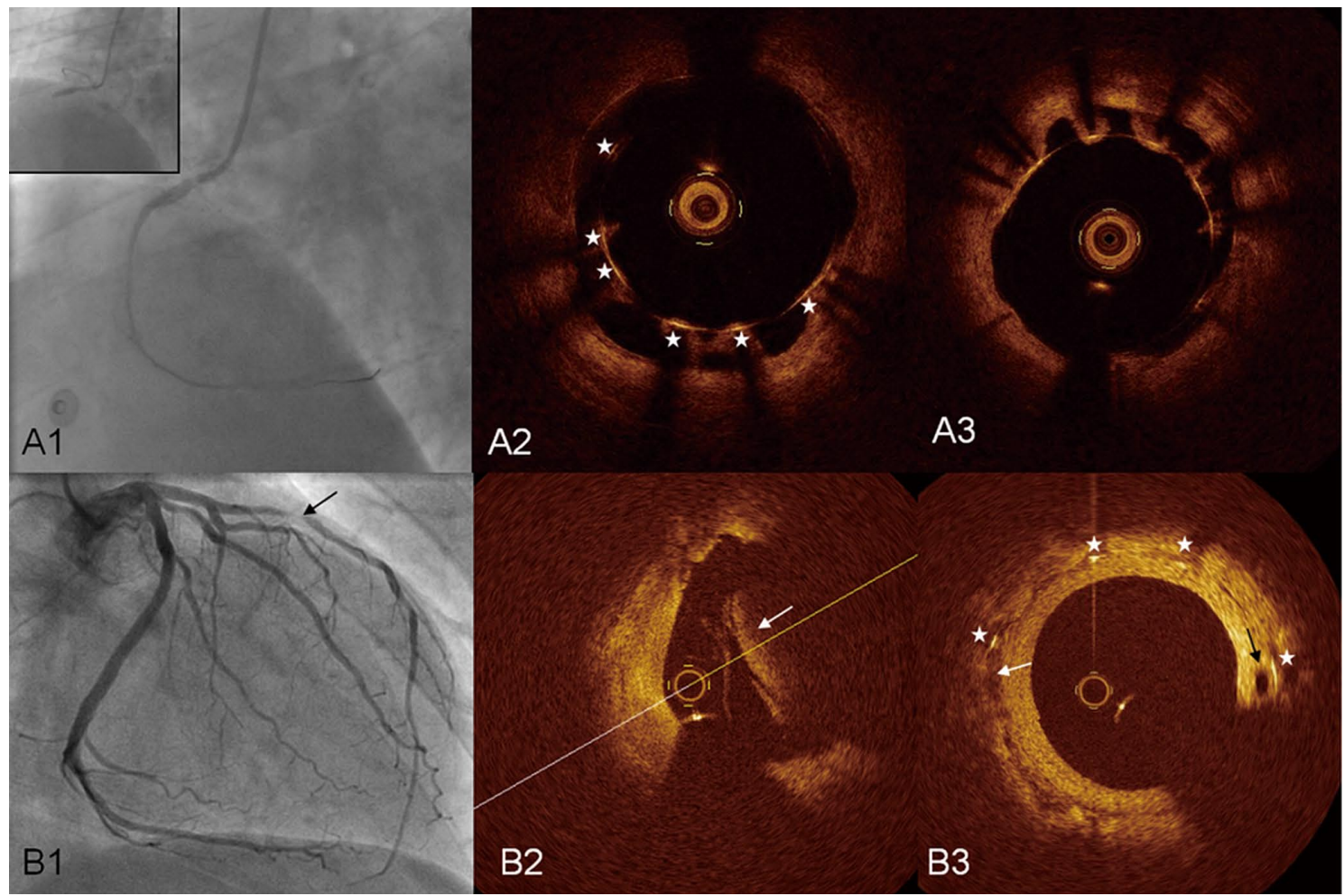

Figure 1. Angiographic and optical coherence tomography (OCT) findings in 2 patients with STEMI late following DES (sirolimus eluting) and BMS implantation. (A1) Total occlusion of the RCA (left upper insert) at the level of a previously implanted DES, and angiographic appearance following thromboaspiration (large image). OCT cross-sections at the level of the thrombosed stent (A2, A3), show multiple uncovered struts $(\star)$, and severe incomplete stent apposition. The latter may be acute (during initial stent implantation for an ACS) or acquired, in which case the dark region behind the struts may be related to vessel remodeling or thrombus dissolution following the previous stent implantation. (B1) Subtotal occlusion of the LAD at the level of a previously implanted BMS. (B2) OCT shows red thrombus (arrow) just proximal to the BMS. (B3) Significant intimal hyperplasia covering all struts ( $\star$ ), a "dark area" just over the struts (arrow), and neovascularization inside neointima (black arrow). STEMI, ST-elevation myocardial infarction; DES, drug-eluting stent; BMS, bare metal stent; RCA, right coronary artery; LAD, left anterior descending.

raphy. In-stent restenosis (ISR) was defined as $\geq 50 \%$ of diameter stenosis (within or $5 \mathrm{~mm}$ proximal or distal to the stent) following thromboaspiration and confirmed by OCT.

\section{OCT Protocol and Analysis}

The M2 and C7-XR Cardiology Imaging OCT Systems (LightLab Imaging, Inc, Westford, MA, USA) with a nonocclusive technique ${ }^{18,19}$ were used. OCT analysis was performed off-line using the LightLab software independently by 2 physicians (V.K., E.M.) who were unaware of the patient's angiographic findings. In case of disagreement regarding OCT qualitative parameters, consensus was reached by a third physician (P.A.D.) who reviewed the corresponding images. OCT images were considered analyzable if the lumen contour could be visualized in $\geq 3$ quadrants. Stent struts were classified as uncovered or covered, and well embedded, protruding or malapposed, as previously described. ${ }^{7}$ Similarly, the protrusion ratio $(\%)$, malapposition ratio (\%), and binary strut coverage (\%) were calculated. ${ }^{20} \mathrm{~A}$ discrete stent was classified as fully covered if every analyzed strut within the stent exhibited neointimal coverage. Thickness $(\mu \mathrm{m})$ of the tissue coverage on the luminal side of each strut was measured and neointimal hyperplasia $[\mathrm{NIH}]$ thickness $(\mu \mathrm{m})$ inside all struts, NIH area $\left(\mathrm{mm}^{2}\right)$, $\mathrm{NIH}$ area $(\%)$ and the heterogeneity score of NIH thickness for each stent were calculated as in previous studies. ${ }^{20}$ Neointimal unevenness score (NUS) and stent eccentricity index, considered to correlate with stent thrombosis, were calculated as described elsewhere. ${ }^{21}$ Luminal area of the reference vessel and stent segment were calculated by manually tracing luminal borders without inclusion of residual thrombus following aspiration, and stent area by manually tracing and connecting stent struts. Percent stenosis was calculated to verify ISR detected on angiography.

Homogeneous signal-rich neointima without signal attenuation ${ }^{17}$ was defined as normal ${ }^{22}$ and lipid, calcification, red and white thrombus, and neovascularization were defined according to objective OCT criteria. $^{22}$ More specifically, heterogeneous signal-poor regions because of marked signal attenuation with diffuse borders were considered as lipid-laden intima. ${ }^{22}$ Possible neointimal tissue rupture was identified as a neointimal cavity communicating with the lumen. ${ }^{14-16}$ Restenosis was defined as $>50 \%$ in-stent area stenosis compared with the native vessel.

\section{Statistical Analysis}

Discrete data are summarized as frequencies and group percentages and continuous data with normal distribution are summarized as mean \pm standard deviation. Student's t-test and 


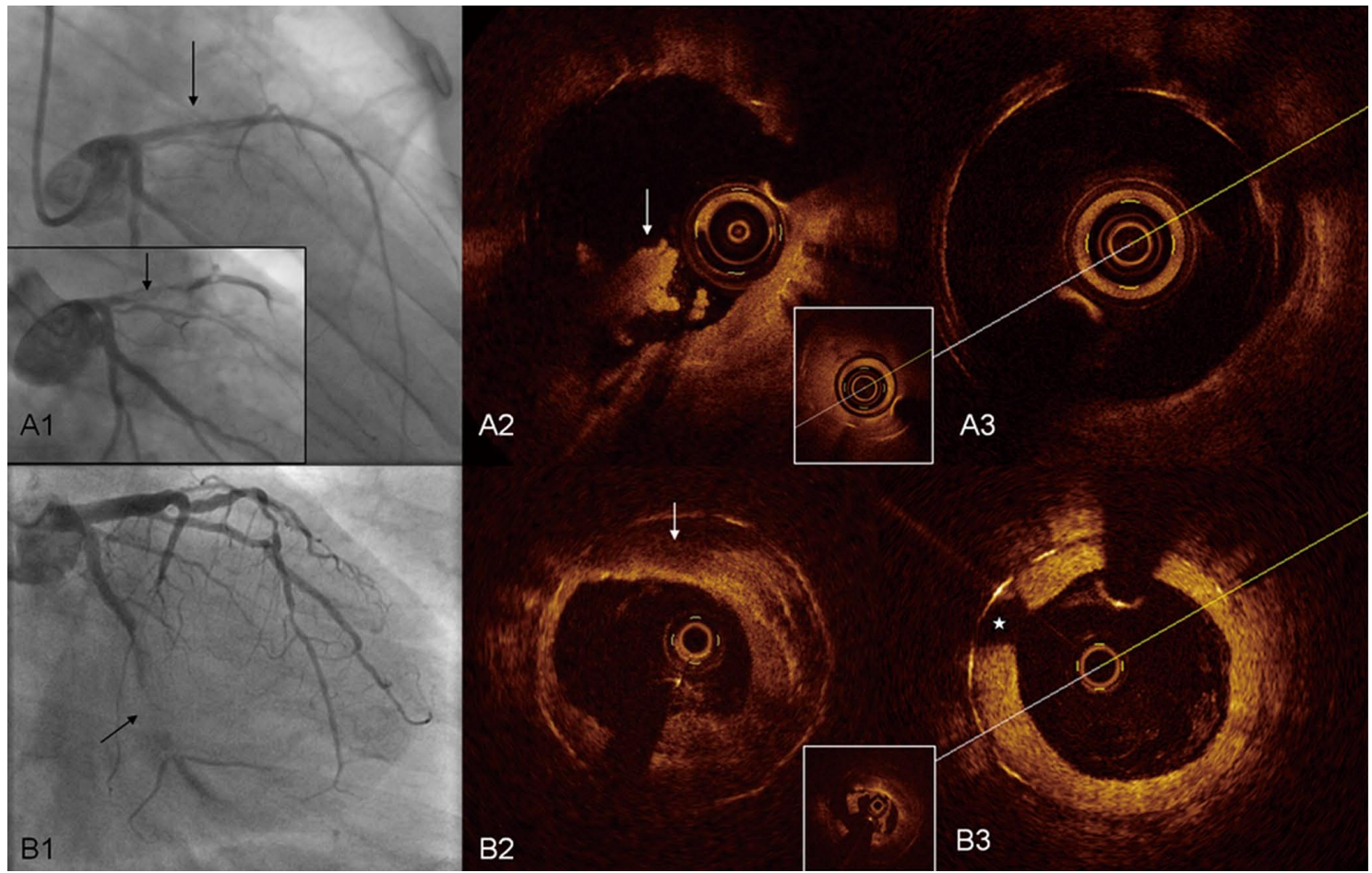

Figure 2. Angiographic and optical coherence tomography (OCT) findings in 2 patients with STEMI late following DES (paclitaxel eluting) and BMS implantation. (A1) Total occlusion of the LAD (left lower insert) at the level of a previously implanted DES, and angiographic appearance following thromboaspiration (large image). OCT cross-sections at the level of the thrombosed stent show white thrombus (A2), and multiple uncovered struts (A3). The insert shows the DES full of thrombus before thromboaspiration. (B1) Thrombosed LCX at the level of a previously implanted BMS. OCT following thromboaspiration in B2 shows significant and uneven intimal hyperplasia with fibrofatty composition (arrow). (B3) Area of possible intimal rupture ( $\star$ ). The insert shows the BMS with thrombus before thromboaspiration. STEMI, ST-elevation myocardial infarction; DES, drug-eluting stent; BMS, bare metal stent; LAD, left anterior descending; LCX, left circumflex.

Fisher's exact test were used to compare continuous and categorical variables, respectively. The interval between initial intervention and follow-up catheterization was compared between the 2 groups with the Mann-Whitney U test because of their skewed distribution. The overall percentages of uncovered, malapposed and protruding struts were compared with ANCOVA models with type of stent as a fixed effect and the number of struts per stent as a covariate, and estimated marginal means (95\% confidence interval $[\mathrm{CI}]$ ) are presented. All tests were 2-tailed and statistical significance was considered for P-values $<0.05$. All statistical analyses were performed using SPSS for Windows (version 16.0; SPSS Inc, Chicago, IL, USA).

This study was performed with the approval of the hospital's ethics committee.

\section{Results}

Of 39 patients presenting with late MI post-stenting, 23 had stent-related MI, 2 were excluded because of hemodynamic instability/renal failure, and 19 consented to an OCT examination; 2 patients were further excluded because of poor OCT image quality, so finally, 17 patients aged $58.9 \pm 8.3$ years were included, 7 with a previously implanted DES and 10 with a BMS. Findings of 1 patient with a BMS were previously described in a case report. ${ }^{16}$ The median time of presentation following initial percutaneous coronary intervention was 50 (3-180) months, with 12 cases (9 BMS, 3 DES) of an MI beyond 12 months post-implantation and 5 cases (1 BMS, 4 DES) between 1 and 12 months post-implantation. The type of DES was paclitaxel $(n=2)$, sirolimus $(n=3)$, biolimus $(n=1)$, and everolimus eluting $(n=1)$. Three of those ( 2 sirolimus, 1 everolimus) were associated with MI $>12$ months post-implantation $(\mathrm{P}=0.4)$. All patients were under dual antiplatelet therapy (clopidogrel $75 \mathrm{mg} /$ day, aspirin $100 \mathrm{mg} /$ day). Table 1 presents the baseline demographic characteristics, the reason for the initial intervention, and initial angiographic anatomy of the studiy population according to the type of stent. Table 2 presents the baseline lesion characteristics. Table 3 presents the results of follow-up coronary angiography because of acute MI, according to the type of stent. Patients with BMS sustained a stent-related non-fatal MI significantly later than those with a DES. Table 4 presents the OCT findings according to the type of stent. In total, 5 BMS and 3 DES were assessed with the time-domain and the remaining (5 BMS, 4 DES) with the frequency-domain OCT system. From the 17 stents (7 DES), 3,434 struts (total length $326 \mathrm{~mm}$ ) were imaged and analyzed. Patients with DES had significantly more uncovered struts per stent, and total cross-sections with $>30 \%$ uncovered struts per stent, and less mean NIH thickness compared with patients with BMS (Figures 1-3). None of the DES was totally covered with neointimal tissue, and the NUS was significantly higher 


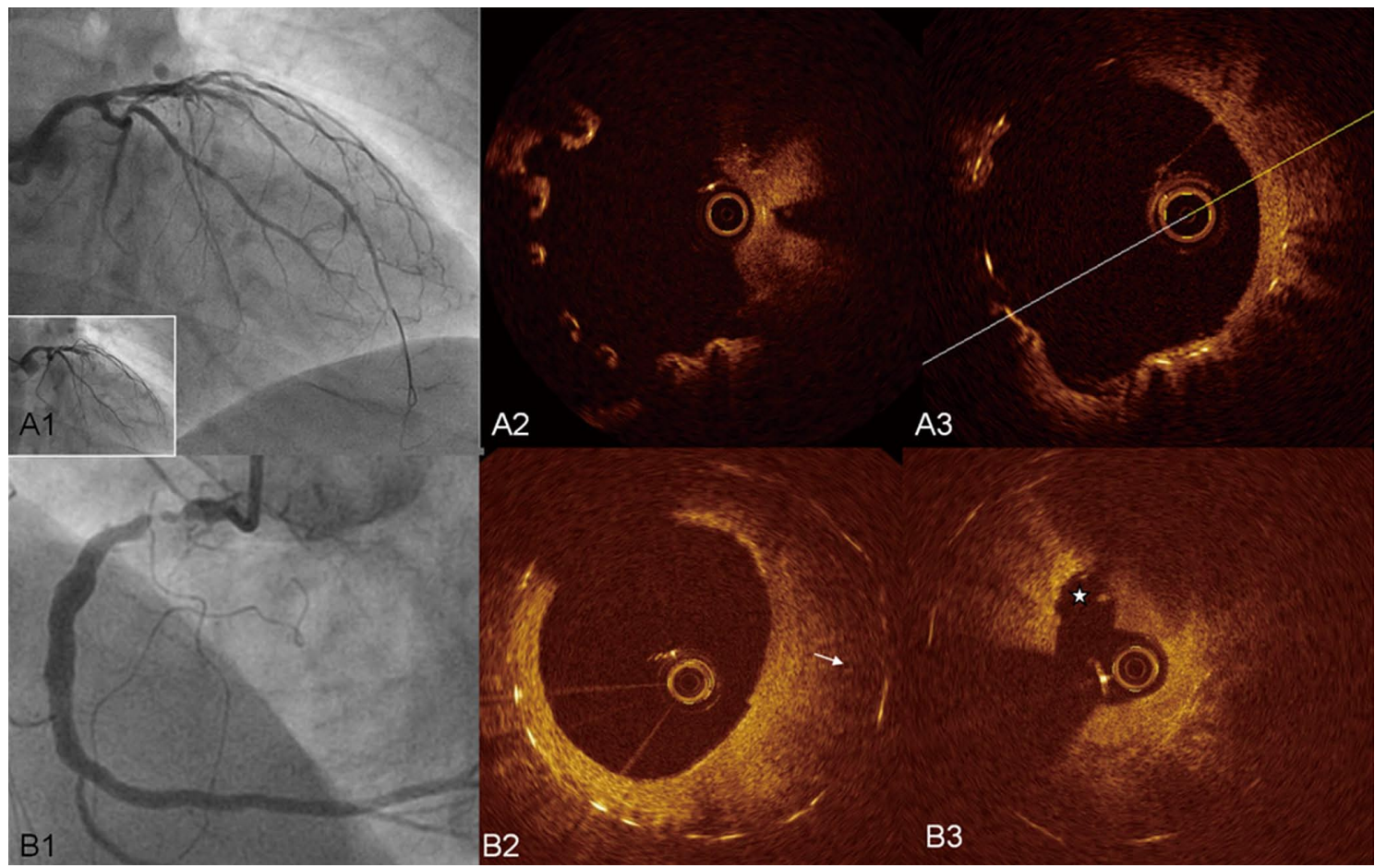

Figure 3. Angiographic and optical coherence tomography (OCT) findings in 2 patients with MI late following DES (STEMI) and BMS (NSTEMI) implantation. (A1) Total occlusion of the LAD (left lower insert) at the level of a previously implanted DES, and angiographic appearance following thromboaspiration (large image). OCT cross-sections at the level of the thrombosed stent show multiple malapposed (A2) and uncovered struts (A2,A3). (B1) Subtotal occlusion of the RCA at the level of a previously implanted BMS. OCT in B2 shows significant and uneven intimal hyperplasia with fibrofatty composition (arrow). (B3) Area of possible intimal rupture $(\star)$. STEMI, ST-elevation myocardial infarction (MI); NSTEMI, non-STEMI; DES, drug-eluting stent; BMS, bare metal stent; LAD, left anterior descending; RCA, right coronary artery.

compared with BMS. Malapposed struts per stent were marginally more frequent in patients with a DES and the malapposition ratio significantly higher (Figures 1-3). The overall percentage of uncovered struts (ANCOVA) was significantly higher $(1.96,95 \% \mathrm{CI} 1.5-2.4$ vs. $0.25-0.1-0.695 \% \mathrm{CI}$, $\mathrm{P}<0.001)$, and that of malapposed struts higher $(0.66,95 \% \mathrm{CI}$ $0.29-1.03$ vs. $0.11,95 \% \mathrm{CI}-0.19-0.4, \mathrm{P}=0.03)$ in DES compared with BMS.

Features of atherosclerosis according to objective OCT criteria such as lipid, neovascularization, or calcification, as well as possible neointimal rupture, were found only in BMS (Figures 1-4). Thromboaspiration was performed in 5/7 DES cases and 4/10 BMS cases before OCT. Thrombus detection with OCT was not different between the 2 groups. Red thrombus was present in $4 \mathrm{BMS}$ and $2 \mathrm{DES}$, and white thrombus in 9 BMS and 4 DES $(\mathrm{P}=0.9)$. In $4(57.1 \%)$ DES cases, but in none of the BMS $(\mathrm{P}=0.08)$, a gap between the struts and vessel wall was detected, (Figure 1). There was total interobserver agreement for variability for lipid, neovascularization, or calcification, but in only 5/7 cases of possible tissue rupture.

\section{Discussion}

Most of the stent-related MI in this series occurred in patients with an acute coronary syndrome (ACS) as the initial indication for intervention. Our main findings are as follows: (1) patients with DES sustained MI significantly earlier compared with those with a BMS and had significantly more uncovered struts, without demonstrating ISR; and (2) patients with BMS had significantly more frequent ISR compared with DES, and demonstrated features of possible neointimal "atherosclerotic transformation" with OCT features of possible neointimal rupture.

Mechanism of Non-Fatal MI Late Following BMS Implantation Angiographic studies have now established that the clinical presentation of BMS restenosis may not be benign and may manifest with thrombosis and MI. ${ }^{23}$ However, the exact mechanisms leading to MI were largely unknown until the advent of high-fidelity intracoronary imaging. Early neointimal tissue within BMS is made up of smooth muscle cells and extracellular matrix. ${ }^{24}$ However a triphasic remodeling pattern of restenosis has been reported, with a possible change in restenotic tissue composition. ${ }^{25}$ Earlier pathologic studies have reported the existence of atherosclerotic features inside BMS very late post-implantation. ${ }^{26}$ More recently, Nakazawa et al in an autopsy series from the CVPath (Gaithersburg, MD, USA) stent registry also reported atherosclerotic lesions inside BMS at a late stage (beyond 24 months). ${ }^{12}$ The variety of neointimal tissue components, and their different densities and orientations, has been hypothesized to be responsible for the heterogeneous appearance with OCT. ${ }^{17}$ Takano et al in a prospective OCT study demonstrated that BMS neointimal tissue may convert to lipid-laden tissue late post-implantation, and lumen 


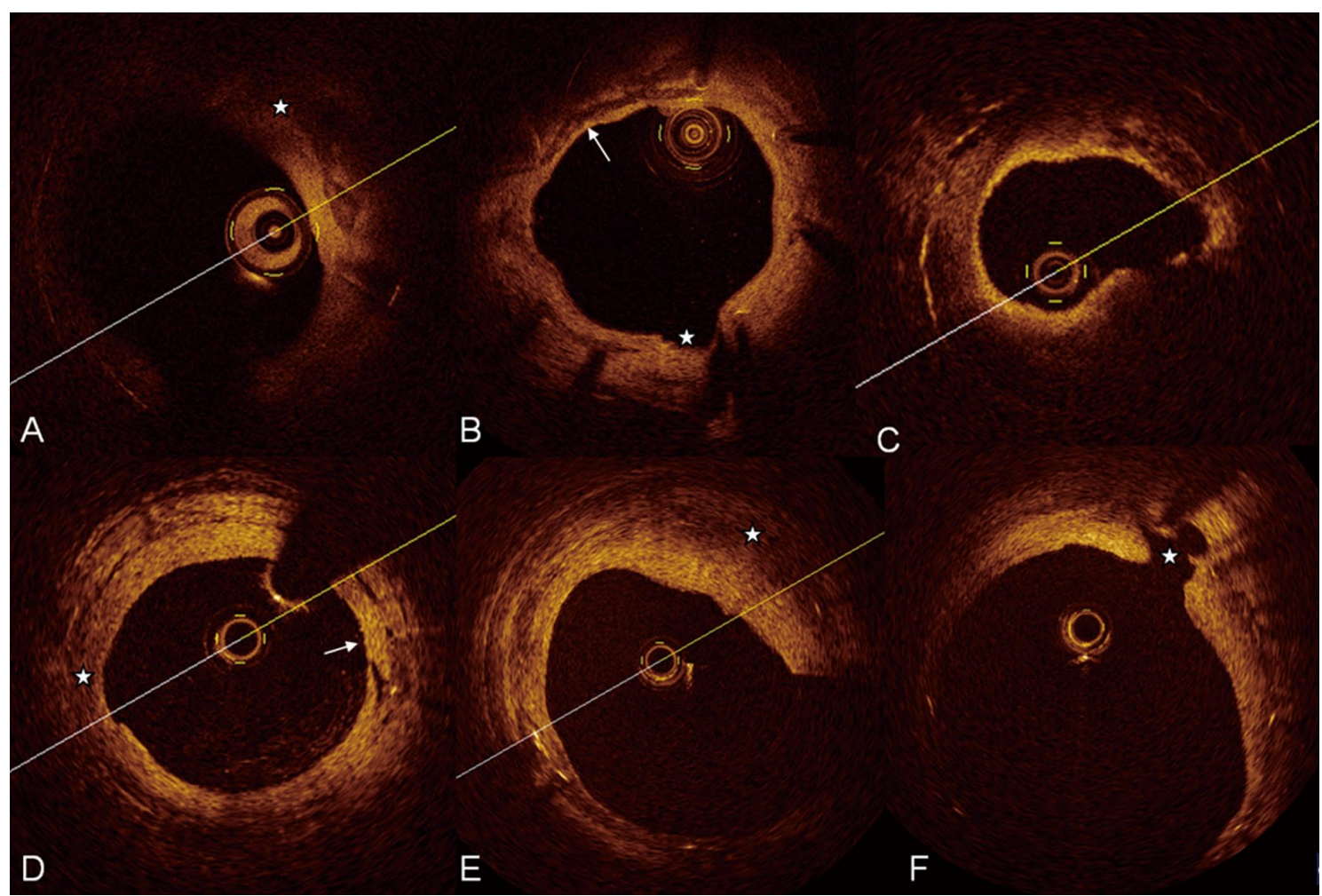

Figure 4. Various OCT findings in patients with late MI following BMS implantation. (A) Fibrolipidic constitution of neointima ( $\star$ ), with calcification (sharply demarcated dark area). (B) Longitudinal calcification of neointima (arrow) with area of possible rupture (asterisk). (C) Circumferential fibrolipidic neointima in a severely restenosed BMS. (D) Fibrolipidic area ( $\star$ ) with area of neovascularization (arrow). (E) Uneven intimal hyperplasia with fibrolipidic area ( $\star$ ). (F) Uneven intimal hyperplasia with area of possible rupture $(\star)$. OCT, optical coherence tomography; DES, drug-eluting stent; BMS, bare metal stent.

narrowing is remarkably increased over those areas. ${ }^{22}$ In the same study the authors reported 4 patients with angiographic restenosis and evidence of lipid-rich neointimal rupture with thrombus. In a very recent report, Hou et al examined with OCT 39 patients with 60 previously implanted BMS presenting with recurrent ischemia, in whom, however, the previously implanted BMS was considered culprit in only 7 patients. ${ }^{15}$ The authors found that $1 / 3$ of BMS demonstrated a lipid-rich plaque, some with evidence of plaque rupture, and a high proportion demonstrating angiographic restenosis and being responsible for unstable angina. Finally, Kashiwagi et al recently reported OCT findings in 4 cases of very late clinical cardiac events over 5 years following BMS implantation. ${ }^{14}$ The authors observed atherosclerotic plaque formation and neovascularization in the stented lesions, and in 2 cases plaque rupture presumably causing the ACS. All these observations were confirmed in our study in which most BMS demonstrated ISR with evidence of lipid-rich neointima, neovascularization, possible neointimal rupture and thrombus (Figures 1-4). Although there is evidence that the status of neointima may differ according to the time of examination, ${ }^{25}$ the vast majority of our BMS-related MIs $(n=9 / 10)$ happened beyond 12 months post-implantation, so we cannot conclude on the possible existence of such a correlation in our series.

Hou et al in their study hypothesized that new atherosclerotic tissue probably grows from the vessel wall through the space between struts into the lumen of the BMS. ${ }^{15}$ Takano et al in their prospective OCT study proposed that persistent microvessels probably advance inside the neointima, building up neovascularization, which may play a key role in atherosclerotic progression and destabilization with intimal disruption and thrombosis. ${ }^{22}$ The fact that more BMS than DES were initially implanted in patients with MI in this study (70\% vs. $28.6 \%$ ), although not statistically significant, may have correlated with inflammation and atherosclerotic change of the BMS neointima, but this needs further study. Whatever the mechanism of this atherosclerotic change, the important message is that it constitutes a potential source of vulnerability and may provoke MI.

Mechanism of Non-Fatal MI Late Following DES Implantation Autopsy studies of DES thrombosis have shown that the most frequent substrate is delayed healing with the existence of uncovered stent struts. ${ }^{4,27}$ Additionally, intravascular ultrasound has shown that DES-LST is associated with incomplete stent apposition, vessel remodeling, and histopathologic evidence of eosinophilic inflammation. ${ }^{2}$ Our study is in accord with those observations, because DES did not demonstrate ISR, and OCT showed significantly more uncovered struts per stent and overall, significantly less NIH thickness compared with corresponding BMS (Figures 1-4). NUS, considered to correlate with LST, ${ }^{21}$ was significantly higher in DES than BMS. We also found a gap between struts and vessel wall only in DES (Figure 1). As more DES were initially implanted because 
of ACS compared with BMS, this gap detected on follow-up OCT might be related to resolution of thrombus trapped behind the stent during initial implantation or to initial incomplete apposition or secondary vessel remodeling.

In contrast to the corresponding BMS, we did not find any OCT features of atherosclerosis such as lipid-rich plaques or neovascularization, and neither did we find any evidence of neointimal tissue rupture in DES. Therefore, we speculate that the prevailing mechanism of MI late following DES implantation is stent thrombosis associated with uncovered stent struts. On the other hand, the reported rate of DES-LST is much lower than the reported rate of uncovered DES struts in prospective studies, underlining the fact that LST is a multifactorial phenomenon. ${ }^{12,28}$ Indeed, Higo et al in their angioscopic study ${ }^{6}$ demonstrated development of "nouveau atherosclerotic plaques" within DES and suggested that this may represent another possible substrate for DES-LST. However, in that prospective study of 57 sirolimus DES at 10 months post-implantation, none of the patients sustained an MI. Additionally, in the autopsy series reported by Nakazawa et al, ${ }^{12}$ although BMS demonstrated significantly more frequent restenosis than DES, atherosclerotic changes were found significantly less frequently in BMS (10\%) compared with DES lesions (35\%). Most importantly, DES demonstrated atherosclerotic changes significantly earlier (since 4 months) compared with BMS (>24 months). Although "nouveau atherosclerosis" inside DES is confirmed by pathologic and angioscopic studies, ${ }^{6,12}$ its contribution to LST and MI is only speculative. Hence, the discrepancy of our results may be because LST and MI following DES implantation are mainly associated with uncovered stent struts.

Another explanation could be that differences in the OCT findings between DES and BMS in our study correlate only with the time interval of the event (MI) post-implantation, as the latter happened significantly later with BMS. However, as mentioned earlier, Nakazawa et al propose an interaction between time and type of stent regarding neointimal composition, with atherosclerotic changes happening inside DES earlier than with BMS. ${ }^{12}$ Still, neither the 4 DES cases associated with an MI between 1 and 12 months nor the 3 DES cases associated with an MI beyond 12 months in our study demonstrated any atherosclerotic features. On the other hand, we did not have any cases of DES-related MI associated with restenotic neointima, which is also not innocent and may manifest with acute MI, ${ }^{1}$ a result probably related to the small number of cases. Without a large series of stent-related MIs demonstrating the full spectrum of the above described OCT features in a variable interval post-implantation, we cannot conclude whether the OCT differences detected between DES and BMS correlate with time, with the specific stent type, or both.

Finally, a very recent report of a DES thrombosis without evidence of any uncovered struts or restenosis, while the patient was on dual antiplatelet therapy, underlines the fact that besides the speculative mechanisms discussed here, we have a long way to go before unraveling the mechanisms of these phenomena. $^{29}$

\section{Study Limitations}

Our observational report, despite being prospective, suffers from a small number of cases $(n=17, \mathrm{DES}=7)$. Additionally, we did not have a case-control design because of the high cost of OCT technology.

Two OCT systems were used (M2 and C7-XR), and because of the slower pullback speed, lower frame rate and smaller field of view of the M2 system, more contrast was needed com- pared with the C7-XR system ( $20 \pm 6$ vs. $8 \pm 2 \mathrm{ml})$. Despite this, we did not record any arrhythmias or cases of contrast-induced nephropathy. Although C7-XR images were clearer, we did not notice any significant difference between the 2 systems in the ability to characterize lesion morphology.

Signal attenuation because of residual thrombus following aspiration may have resulted in a reduced number of analyzable struts, especially in DES. Thromboaspiration catheters were used before OCT when the thrombotic burden was large, and may have caused tissue trauma. Therefore, tissue characterization mainly regarding neointimal rupture, and planimetric data should be viewed cautiously. However, DES cases did not demonstrate tissue rupture. Additionally, thromboaspiration in combination with more sensitive detection of thrombus with OCT compared with angiography, may explain the different frequency of thrombus detection between DES and BMS. The ANCOVA models produced estimates with relatively large $\mathrm{CI}$, probably because of the relatively small number of patients included.

As there are no OCT criteria validated with histology for the identification of neointimal components, tissue characterization with objective OCT criteria as applied in native atherosclerosis is speculative. ${ }^{17}$ However, others have used similar criteria for neointimal tissue characterization. ${ }^{22}$ There was lack of unanimity regarding possible tissue rupture in 2 of 7 cases. This type of finding needs further clarification and standardization.

\section{Clinical Relevance and Future Perspectives}

Our population is not representative of all patients with MI following stenting, as patients who died before angiography, sustained a "silent" MI, or were treated conservatively were not included, and we cannot comment on the potential mechanisms of MI associated with DES restenosis as we did not encounter such cases. For these reasons, our P-values regarding OCT differences between BMS- and DES-related MI should be interpreted with caution, and are only indicative. However, it is evident that the high resolution of OCT makes it an excellent tool for studying stent-related clinical events. Larger pathologic, angioscopic, and OCT studies, or the establishment of a relevant OCT registry, may shed more light on the relative contribution of uncovered struts, new atherosclerotic plaques, and DES or BMS restenotic tissue in the mechanisms of LST and MI following stent implantation, and/or the potential effects of established atherosclerotic-modifying therapies such as statins, on this type of neointimal "atherosclerotic transformation".

\section{Conclusions}

The mechanisms of non-fatal stent-related acute MI late following stent implantation may differ between DES and BMS. Delayed healing may be the prevailing mechanisms associated with DES-related MI, whereas neointimal tissue growth with atherosclerotic transformation and subsequent rupture may be more frequently associated with BMS-related MI.

\section{References}

1. Alexopoulos D, Xanthopoulou I, Davlouros P, Damelou A, Mazarakis A, Chiladakis J, et al. Mechanisms of nonfatal acute myocardial infarction late after stent implantation: The relative impact of disease progression, stent restenosis, and stent thrombosis. Am Heart J 2010; 159: 439-445.

2. Cook S, Ladich E, Nakazawa G, Eshtehardi P, Neidhart M, Vogel R, et al. Correlation of intravascular ultrasound findings with histopathological analysis of thrombus aspirates in patients with very late 
drug-eluting stent thrombosis. Circulation 2009; 120: 391-399.

3. Shite J. Delayed neointimalization on drug-eluting stents: Speculation from optical coherence tomography. Circ J 2009; 73: 2210 2211.

4. Finn AV, Joner M, Nakazawa G, Kolodgie F, Newell J, John MC, et al. Pathological correlates of late drug-eluting stent thrombosis: Strut coverage as a marker of endothelialization. Circulation 2007; 115: $2435-2441$.

5. Finn AV, Nakazawa G, Joner M, Kolodgie FD, Mont EK, Gold HK, et al. Vascular responses to drug eluting stents: Importance of delayed healing. Arterioscler Thromb Vasc Biol 2007; 27: 1500-1510.

6. Higo T, Ueda Y, Oyabu J, Okada K, Nishio M, Hirata A, et al. Atherosclerotic and thrombogenic neointima formed over sirolimus drug-eluting stent: An angioscopic study. JACC Cardiovasc Imaging 2009; 2: 616-624.

7. Davlouros P, Nikokiris G, Karantalis V, Mavronasiou E, Xanthopoulou I, Damelou A, et al. Neointimal coverage and stent strut apposition six months after implantation of a paclitaxel eluting stent in acute coronary syndromes: An optical coherence tomography study. Int $J$ Cardiol 2010 [E-pub ahead of print].

8. Ishigami K, Uemura S, Morikawa Y, Soeda T, Okayama S, Nishida T, et al. Long-term follow-up of neointimal coverage of sirolimus-eluting stents: Evaluation with optical coherence tomography. Circ J 2009; 73: $2300-2307$

9. Katoh H, Shite J, Shinke T, Matsumoto D, Tanino Y, Ogasawara D, et al. Delayed neointimalization on sirolimus-eluting stents: 6-month and 12-month follow up by optical coherence tomography. Circ J 2009; 73: 1033-1037.

10. Kim JS, Kim TH, Fan C, Lee JM, Kim W, Ko YG, et al. Comparison of neointimal coverage of sirolimus-eluting stents and paclitaxeleluting stents using optical coherence tomography at 9 months after implantation. Circ J 2010; 74: 320-326.

11. Serruys PW, Daemen J. Are drug-eluting stents associated with a higher rate of late thrombosis than bare metal stents? Late stent thrombosis: A nuisance in both bare metal and drug-eluting stents. Circulation 2007; 115: 1433-1439; discussion 1439.

12. Nakazawa G, Vorpahl M, Finn AV, Narula J, Virmani R. One step forward and two steps back with drug-eluting-stents: From preventing restenosis to causing late thrombosis and nouveau atherosclerosis. JACC Cardiovasc Imaging 2009; 2: 625-628.

13. Ramcharitar S, Garcia-Garcia HM, Nakazawa G, Kukreja N, Ligthart J, Virmani R, et al. Ultrasonic and pathological evidence of a neointimal plaque rupture in patients with bare metal stents. EuroIntervention 2007; 3: 290-291.

14. Kashiwagi M, Kitabata H, Tanaka A, Okochi K, Ishibashi K, Komukai $\mathrm{K}$, et al. Very late clinical cardiac event after bms implantation: In vivo optical coherence tomography examination. JACC Cardiovasc Imaging 2010; 3: 525-527.

15. Hou J, Qi H, Zhang M, Ma L, Liu H, Han Z, et al. Development of lipid-rich plaque inside bare metal stent: Possible mechanism of late stent thrombosis? An optical coherence tomography study. Heart 2010; 96: $1187-1190$.
16. Davlouros PA, Karantalis V, Mavronassiou E, Alexopoulos D. Neointimal tissue rupture as a mechanism of myocardial infarction very late following implantation of bare metal stents: Insights from optical coherence tomography. Int J Cardiol 2011; 148: 348-349.

17. Gonzalo N, Serruys PW, Okamura T, van Beusekom HM, GarciaGarcia HM, van Soest G, et al. Optical coherence tomography patterns of stent restenosis. Am Heart J 2009; 158: 284-293.

18. Kataiwa H, Tanaka A, Kitabata H, Imanishi T, Akasaka T. Safety and usefulness of non-occlusion image acquisition technique for optical coherence tomography. Circ J 2008; 72: 1536-1537.

19. Prati F, Cera M, Ramazzotti V, Imola F, Giudice R, Giudice M, et al. From bench to bedside: A novel technique of acquiring oct images. Circ J 2008; 72: 839-843.

20. Kim JS, Fan C, Choi D, Jang IK, Lee JM, Kim TH, et al. Different patterns of neointimal coverage between acute coronary syndrome and stable angina after various types of drug-eluting stents implantation; 9-month follow-up optical coherence tomography study. Int $J$ Cardiol 2009; 146: 341-346.

21. Otake H, Shite J, Ako J, Shinke T, Tanino Y, Ogasawara D, et al. Local determinants of thrombus formation following sirolimus-eluting stent implantation assessed by optical coherence tomography. JACC Cardiovasc Interv 2009; 2: 459-466.

22. Takano M, Yamamoto M, Inami S, Murakami D, Ohba T, Seino Y, et al. Appearance of lipid-laden intima and neovascularization after implantation of bare-metal stents extended late-phase observation by intracoronary optical coherence tomography. J Am Coll Cardiol 2009; 55: 26-32.

23. Doyle B, Rihal CS, O'Sullivan CJ, Lennon RJ, Wiste HJ, Bell M, et al. Outcomes of stent thrombosis and restenosis during extended follow-up of patients treated with bare-metal coronary stents. Circulation 2007; 116: $2391-2398$.

24. Farb A, Kolodgie FD, Hwang JY, Burke AP, Tefera K, Weber DK, et al. Extracellular matrix changes in stented human coronary arteries. Circulation 2004; 110: 940-947.

25. Inoue K, Abe K, Ando K, Shirai S, Nishiyama K, Nakanishi M, et al Pathological analyses of long-term intracoronary palmaz-schatz stenting; is its efficacy permanent? Cardiovasc Pathol 2004; 13: 109115.

26. Hasegawa K, Tamai H, Kyo E, Kosuga K, Ikeguchi S, Hata T, et al. Histopathological findings of new in-stent lesions developed beyond five years. Catheter Cardiovasc Interv 2006; 68: 554-558.

27. Joner M, Finn AV, Farb A, Mont EK, Kolodgie FD, Ladich E, et al. Pathology of drug-eluting stents in humans: Delayed healing and late thrombotic risk. J Am Coll Cardiol 2006; 48: 193-202.

28. Iakovou I, Schmidt T, Bonizzoni E, Ge L, Sangiorgi GM, Stankovic $\mathrm{G}$, et al. Incidence, predictors, and outcome of thrombosis after successful implantation of drug-eluting stents. JAMA 2005; 293: 21262130.

29. Nishiguchi T, Kitabata H, Tanaka A, Kataiwa H, Ishibashi K, Komukai $\mathrm{K}$, et al. Very late stent thrombosis after drug-eluting stent in segment with neointimal tissue coverage. JACC Cardiovasc Imaging 2010; 3: 445-446. 